\title{
THE CLASSICAL IRRATIONALITY PROBLEM FOR $T$-FRACTIONS
}

\author{
R. M. HOVSTAD
}

(Communicated by Paul S. Muhly)

\begin{abstract}
Necessary and sufficient conditions are given for a $T$-fraction to correspond to a rational function.
\end{abstract}

\section{INTRODUCTION}

The subject for study in this paper is the general $T$-fraction

$$
\mathfrak{K}_{k=1}^{\infty} \frac{z}{e_{k}+d_{k} z},
$$

where $e_{k} \neq 0$ and $d_{k}$ for $k \geq 1$ are complex numbers. The continued fraction (1.1) corresponds to a uniquely determined formal power series

$$
g_{1}(z)=\frac{z}{e_{1}}+\cdots
$$

at $z=0$. For background to $T$-fractions and correspondence we refer to the recent book [4] by Jones and Thron.

The problem discussed in this paper is what kind of restrictions must be imposed on (1.1) so we can conclude that (1.2) is the power series of a rational function. This problem is mentioned in the classic book [5, p. 174] and recent contributions to this problem area by Jefferson, Hag and Waadeland, are reported and commented in [4, p. 380]. See also [1-3, 7]. In the next paragraph we give a solution of this problem in the form of necessary and sufficient conditions involving a polynomial sequence satisfying certain properties. This provides us with a device which makes it possible to track down all possible types of rationality in (1.2). A corollary is given where the simplest type is treated explicitly.

The method used in the present treatment is founded on the concept of tails. We call

$$
\stackrel{\mathrm{K}}{i=k}_{e_{i}+d_{i} z}
$$

Received by the editors August 12, 1988 and, in revised form, September 18, 1989.

1980 Mathematics Subject Classification (1985 Revision). Primary 30B70. 
for $k \geq 1$ the tails of (1.1). We notice that (1.3) corresponds to a uniquely determined formal power series

$$
g_{k}(z)=\frac{z}{e_{k}}+\cdots
$$

at $z=0$ for $k \geq 1$. Tails have recently been in focus in the theory of continued fractions. See especially Waadeland's paper [8]. However, the present author's use of tails originated independently, and later, in an analysis of the role of the Euclidean algorithm in the classical theory of irrational numbers by the aid of continued fractions. See $[5$, p. 56] and $[6$, p. 22].

\section{RATIONALITY}

The solution of the problem is given in the following theorem. We note that $\operatorname{deg} p \geq 0$ means the degree of a polynomial $p$.

Theorem. The continued fraction (1.1) corresponds to a rational function at $z=0$ if and only if there exist an integer $q \geq 0$ and a sequence of polynomials $\left\{p_{k}\right\}_{k \geq 0}$ with the following three properties

$$
\begin{aligned}
& \operatorname{deg} p_{k}=k+q \\
& p_{k}(z)=a_{k} z^{k}+\cdots \quad\left(a_{k} \neq 0\right) \\
& z p_{k-1}(z)=\left(e_{k}+d_{k} z\right) p_{k}(z)+p_{k+1}(z)
\end{aligned}
$$

for $k$ sufficiently large. In case of rationality we have that (1.3) corresponds to

$$
\frac{p_{k}(z)}{p_{k-1}(z)}=\frac{z}{e_{k}}+\cdots
$$

at $z=0$ for $k$ sufficiently large.

Proof. First, suppose that (1.1) corresponds to a rational function $p_{1}(z) / p_{0}(z)$, where $p_{0}(z)$ and $p_{1}(z)$ are polynomials. Obviously we can choose $p_{0}(z)=$ $1+\cdots$ and $p_{1}(z)=z / e_{1}+\cdots$. Define the sequence $\left\{p_{k}\right\}_{k \geq 0}$ of formal power series by the formal identity

$$
p_{k}(z)=g_{k}(z) p_{k-1}(z)
$$

for $k \geq 2$. We notice that (2.5) is also true formally for $k=1$ and the polynomials $p_{0}$ and $p_{1}$ can be considered as formal power series where only a finite number of the coefficients in the expansions are nonzero. From (1.4) and (2.5) we conclude by induction that

$$
p_{k}(z)=a_{k} z^{k}+\cdots
$$

for $k \geq 0$ where $e_{k} a_{k}=a_{k-1}$ for $k \geq 1$ with $a_{0}=1$. From (1.3) and (1.4) we see that

$$
g_{k}(z)=\frac{z}{e_{k}+d_{k} z+g_{k+1}(z)}
$$


formally for $k \geq 1$. The formal identities (2.5) and (2.7) further give that

$$
z p_{k-1}(z)=\left(e_{k}+d_{k} z\right) p_{k}(z)+p_{k+1}(z)
$$

formally for $k \geq 1$. The identity (2.8) is a relation involving formal power series such that $p_{0}$ and $p_{1}$ have only a finite number of nonzero coefficients in their expansions. By induction we then see from (2.8) that $p_{k}$ for all $k \geq 0$ has only a finite number of nonzero coefficients in the expansion. This means that $\left\{p_{k}\right\}_{k \geq 0}$ can be considered a polynomial sequence. This is the polynomial sequence in the theorem. From (2.6) we therefore conclude that $\operatorname{deg} p_{k} \geq k$ for $k \geq 0$. Further from (2.8) we find that $\operatorname{deg} p_{k+1} \leq \max \left(\operatorname{deg} p_{k}, \operatorname{deg} p_{k-1}\right)+1$ for $k \geq 1$. Thus

$$
0<\max \left(\operatorname{deg} p_{k+1}, \operatorname{deg} p_{k}\right)-k \leq \max \left(\operatorname{deg} p_{k}, \operatorname{deg} p_{k-1}\right)-(k-1)
$$

for $k \geq 1$. Clearly we cannot have strict inequality in the right inequality of (2.9) for infinitely many values of $k$ since this would produce a descending infinite sequence of positive integers which is clearly impossible. Thus we have

$$
\max \left(\operatorname{deg} p_{k+1}, \operatorname{deg} p_{k}\right)=\max \left(\operatorname{deg} p_{k}, \operatorname{deg} p_{k-1}\right)+1
$$

for $k \geq k_{0} \geq 1$, where $k_{0}$ is a suitable chosen integer. Let $n \geq k_{0}$ be a fixed integer. We discriminate between two cases. The first case is when $\operatorname{deg} p_{n} \geq \operatorname{deg} p_{n-1}$ and the second case is when $\operatorname{deg} p_{n}<\operatorname{deg} p_{n-1}$. Consider the first case. Then from (2.10) we find that $\max \left(\operatorname{deg} p_{n+1}, \operatorname{deg} p_{n}\right)=$ $\operatorname{deg} p_{n}+1$ and therefore $\operatorname{deg} p_{n+1}=\operatorname{deg} p_{n}+1$. Next consider the second case. We find then from (2.10) that $\max \left(\operatorname{deg} p_{n+1}, \operatorname{deg} p_{n}\right)=\operatorname{deg} p_{n-1}+1$ and therefore $\operatorname{deg} p_{n+1}=\operatorname{deg} p_{n-1}+1>\operatorname{deg} p_{n}$. Again using (2.10) we find that $\max \left(\operatorname{deg} p_{n+2}, \operatorname{deg} p_{n+1}\right)=\operatorname{deg} p_{n+1}+1$ and therefore $\operatorname{deg} p_{n+2}=\operatorname{deg} p_{n+1}+1$. Thus in both cases we arrive at the existence of an integer $k \geq k_{0}+1$ such that $\operatorname{deg} p_{k}=\operatorname{deg} p_{k-1}+1$. Consider therefore some integer $k \geq k_{0}+1$ where $\operatorname{deg} p_{k}=\operatorname{deg} p_{k-1}+1$. From (2.10) we conclude that $\max \left(\operatorname{deg} p_{k+1}, \operatorname{deg} p_{k}\right)=$ $\operatorname{deg} p_{k}+1$ and therefore $\operatorname{deg} p_{k+1}=\operatorname{deg} p_{k}+1$. In this way we continue and clearly we have

$$
\operatorname{deg} p_{k}=\operatorname{deg} p_{k-1}+1
$$

for $k$ sufficiently large. From (2.11) we conclude that $\operatorname{deg} p_{k}-k=q \geq 0$ is constant for $k$ sufficiently large. Thus we have shown the existence of an integer $q \geq 0$ (this is the number $q$ in the theorem) such that

$$
\operatorname{deg} p_{k}=k+q
$$

for $k$ sufficiently large and condition $(2.1)$ in the theorem is proved. In addition, conditions (2.2) and (2.3) are easily concluded from (2.6) and (2.8).

Second, let the conditions $(2.1)-(2.3)$ in the theorem be fulfilled for a sequence $\left\{p_{k}\right\}_{k \geq 0}$ of polynomials and a fixed integer $q \geq 0$. Suppose that conditions (2.1)-(2.3) are true for $k \geq h_{0} \geq 1$, where $h_{0}$ is a suitable integer. Then certainly

$$
z p_{k-1}(z)=\left(e_{k}+d_{k} z\right) p_{k}(z)+p_{k+1}(z)
$$


for $k \geq h_{0}+1$. Since for $k \geq h_{0}, p_{k}(z)$ can be written $p_{k}(z)=z^{k} f_{k}(z)$ where

$$
f_{k}(z)=a_{k}+\cdots
$$

for $k \geq h_{0}$ is a polynomial of degree $q$, we see from (2.13) that

$$
\frac{z f_{k}(z)}{f_{k-1}(z)}=\frac{z}{e_{k}+d_{k} z+z f_{k+1}(z) / f_{k}(z)}
$$

for $k \geq h_{0}+1$. We will now show that

$$
\underset{k=h_{0}+1}{\mathrm{~K}} \frac{z}{e_{k}+d_{k} z}
$$

corresponds to the rational function

$$
\frac{z f_{h_{0}+1}(z)}{f_{h_{0}}(z)}=\frac{a_{h_{0}+1}}{a_{h_{0}}} z+\cdots
$$

at $z=0$. To this end we consider the usual approximants of (2.16) denoted by $A_{n}(z) / B_{n}(z)$ in the standard notation (see for example the book [4]). We find

$$
\begin{aligned}
& \left|\frac{z f_{h_{0}+1}(z)}{f_{h_{0}}(z)}-\frac{A_{n}(z)}{B_{n}(z)}\right| \\
& \quad=\left|\frac{f_{h_{0}+n}(z) A_{n}(z)+z f_{h_{0}+n+1}(z) A_{n-1}(z)}{f_{h_{0}+n}(z) B_{n}(z)+z f_{h_{0}+n+1}(z) B_{n-1}(z)}-\frac{A_{n}(z)}{B_{n}(z)}\right| \\
& =\frac{\left|f_{h_{0}+n+1}(z)\right||z|^{n+1}}{\left|B_{n}(z)\left(f_{h_{0}+n}(z) B_{n}(z)+z f_{h_{0}+n+1}(z) B_{n-1}(z)\right)\right|}
\end{aligned}
$$

for $n \geq 0$. The equations in (2.18) are standard. See for example [4, p. 20]. It should however be pointed out that the first equality in (2.18) arises from repeated application of (2.15). From $(2.14),(2.18)$ and the fact that $B_{n}(0) \neq 0$ we see that (2.16) corresponds to (2.17) at $z=0$. This gives us as a conclusion that also (1.1) corresponds to a rational function at $z=0$.

Finally, we notice that $(2.4)$ in the theorem is established in the light of (2.17) and the proof is completed (remember that $a_{h_{0}}=e_{h_{0}+1} a_{h_{0}+1}$ follows from (2.13)).

The integer $q \geq 0$ in the theorem is important for practical purposes. According to increasing values of $q$ we can trace all possible types of rational correspondences of (1.1). We treat the simplest type where $q=0$ in the following corollary.

Corollary (case $q=0$ ). If the continued fraction (1.1) obeys

$$
d_{k}+\frac{1}{e_{k+1}}=0
$$

for sufficiently large $k$, then (1.1) corresponds to a rational function at $z=0$. More explicitly we have that

$$
\underset{i=k}{\mathrm{~K}} \frac{z}{e_{i}-z / e_{i+1}}
$$

corresponds to $z / e_{k}$ at $z=0$ for sufficiently large $k$. 
Proof. Let $q=0$ in the theorem. Consider a sequence $\left\{p_{k}\right\}_{k \geq 0}$ of polynomials where

$$
p_{k}(z)=a_{k} z^{k}
$$

with $a_{k} \neq 0$ for $k$ sufficiently large. Clearly conditions (2.1) and (2.2) in the theorem are fulfilled. After having introduced (2.21) in the condition (2.3) in the theorem, this condition reads

$$
a_{k-1} z^{k}=a_{k}\left(e_{k}+d_{k} z\right) z^{k}+a_{k+1} z^{k+1}
$$

for $k$ sufficiently large. Simple calculation shows that (2.22) is equivalent to $e_{k} a_{k}=a_{k-1}$ and $d_{k} a_{k}+a_{k+1}=0$ for $k$ sufficiently large. This implies (2.19). On the other hand if (2.19) is true we clearly can choose the numbers $a_{k}$ in (2.21) such that $e_{k} a_{k}=a_{k-1}$ for $k$ sufficiently large, and this in turn implies $d_{k} a_{k}+a_{k+1}=0$ for $k$ sufficiently large. Thus (2.19) is necessary and sufficient for the continued fraction (1.1) to correspond to a rational function at $z=0$ in case $q=0$. In addition, statement (2.4) in the theorem gives us (2.20). All this implies the corollary.

Choosing $e_{k}=1$ and $d_{k}=-1$ for $k \geq 1$ in the corollary we have that the continued fraction

$$
\stackrel{K}{k=1}_{1-z} \frac{z}{1-z}
$$

corresponds to the rational function $z$ at $z=0$. The example (2.23) is well known in the literature. See for example [4, p. 146]. Also the corollary in general is well known. See [4, Theorem 7.20], [7, Observation 3, p. 29], and [9, $\S 3]$.

We can continue to find explicit formulas of the type (2.19) for the cases where $q \geq 1$. The formulas will get more and more involved for increasing values of $q$. This illustrates the complex nature of the problem of rational correspondence of general $T$-fractions. This implies that it is difficult to decide from the theorem whether a given $T$-fraction corresponds to a rational function or not, in spite of the fact that explicit conditions for all types of rational correspondences can in principle be obtained by following the calculations necessitated by the theorem and the difficulties just mentioned are only of a technical nature.

\section{ACKNOWLEDGMENT}

The author would like to thank the referee for the comments on the original version of this paper. 


\section{REFERENCES}

1. K. Hag, A theorem on $T$-fractions corresponding to a rational function, Proc. Amer. Math. Soc. 25 (1970), 247-253.

2. __ A convergence theorem for Limitärperiodisch T-fractions of rational functions, Proc. Amer. Math. Soc. 32 (1972), 491-496.

3. T. H. Jefferson, Some additional properties of $T$-fractions, $\mathrm{Ph}$. $\mathrm{D}$. thesis, University of Colorado, Boulder, Colorado, 1969.

4. W. B. Jones and W. J. Thron, Continued fractions, Analytic theory and applications, Addison-Wesley, Reading, MA, 1980.

5. O. Perron, Die Lehre von den Kettenbrüchen, Band II, 3. Aufl., Teubner, Stuttgart, 1957.

6. __ Die Lehre von den Kettenbrüchen, Band I, 3. Aufl, Teubner, Stuttgart, 1954.

7. H. Waadeland, Some properties of general T-fractions, Univ. i Trondheim, Matematisk Institutt (NLHT), Trondheim, 1978.

8. __ Tales about tails, Proc. Amer. Math. Soc. 90 (1984), 57-64.

9. - , General T-expansions of bounded functions, Dept. of Math., Trondheim, no. 2, 1983.

NAKkVES Vei 5 L1020, 0670 Oslo 6, NoRWAy 